International Journal of Applied Mathematics

Volume 31 No. $5 \quad 2018,647-669$

ISSN: 1311-1728 (printed version); ISSN: 1314-8060 (on-line version)

doi: http://dx.doi.org/10.12732/ijam.v31i5.10

\title{
NUMERICAL SCHEMES FOR SOLVING VOLTERRA INTEGRAL EQUATIONS WITH CARLEMAN KERNEL
}

\author{
Wala Draidi ${ }^{1}$, Naji Qatanani ${ }^{2} \S$ \\ ${ }^{1,2}$ Department of Mathematics \\ An-Najah National University \\ Nablus, PALESTINE
}

\begin{abstract}
Three numerical schemes, namely: Toeplitz Matrix meth-od, Product Nystrom method and Sinc-Collocation method have been proposed and implemented to give an approximate solution of the linear Volterra integral equation of the second kind with Carleman kernel. To display the validity and acceptability of the numerical methods, two illustrative examples with known exact solution are presented. Numerical results show clearly that the convergence and accuracy of these schemes are in a good agreement with the exact solution. Moreover, it is worth pointing out that the Nystrom and Toeplitz matrix schemes are more efficient in comparison with the sinc-collocation method.
\end{abstract}

AMS Subject Classification: 45D05, 65R20, 68Wxx

Key Words: Toeplitz matrix method, product Nystrom method, sinc-collocation method

\section{Introduction}

In recent years, singular integral equations have attracted the attention of many scientists and researchers due to their wide range of applications in science and technology. Many problems related to mathematical physics, engineering, theory of elasticity and the mixed problems of continuous media lead to integral equations of the first or second kind [7], [9].

Carleman was the first pioneer to realize the importance of the Fredholm integral equation and its applications [14]. The importance of Carleman kernel came from the work of Arytiunian [8] who has shown that the contact problem

Received: July 5, 2018

(c) 2018 Academic Publications

${ }^{\S}$ Correspondence author 
of nonlinear theory of elasticity in the first approximation reduces to a Fredholm integral equation of the first kind with Carleman kernel. Kreins technique [5] is used to find the relationship between integral which has Carleman kernel and integral with logarithmic kernel.

Analytical solutions are only available for solving simple problems involving Carleman kernel (for more details see Linz [22] and Muskhelishvili [23]). On the other hand, numerical methods play a very important role nowadays in solving singular integral equations.

Abdalkhani [1] obtained a numerical solution of the nonlinear Volterra integral equation with Carleman kernel. Abdou, in his work [2], [3], [4] used a series in the Legendre polynomials form to obtain the solution of FredholmVolterra integral equation of the second kind under certain conditions. In [16], Guoqiang used the extrapolation method of iterated collocation solution of two dimensional nonlinear Volterra integral equation. Brunner [10], [11] has used the sinc-collocation method to solve Fredholm integral equation of the second kind with weakly singular kernel. On the other hand, Okayama [24] implemented the sinc-collocation method to find an approximate solution to weakly singular Fredholm integral equations of the second kind.

The Laplace Adomian decomposition method has been introduced by Hendi and Al-Hazmi [17], [18] to obtain the solution of nonlinear Volterra integral equation with weakly kernels. Khan and Bakodah [20] have realized the importance of the Adomian Decomposition Method and its Modification to solve nonlinear Abel's integral equation. In [15], Graham applied the Galerkin method to obtain the solution of singular integral equations of the second kind. Furthermore, Orsi [25] has used product Nystrom as numerical method for the same purpose. In [19], Kauthen applied a linear multistep to obtain approximate solution of singular perturbed Volterra integral equations, whereas Kilbas and Saigo [21] used an asymptotic method to get the solution of the nonlinear Abel's integral equation.

Tong and Chew [26], [27], [28] used Nystrom scheme for electromagnetic scattering by arbitrarily shaped surfaces, 2D open structures and complex material bodies. Further, Tong, Qian and Chew [29] considered Nystrom scheme of volume integral equations for electromagnetic scattering by $3 \mathrm{D}$ penetrable objects. Draidi [12] in her thesis investigated the numerical handling of integral equations with Carleman kernel.

In this work, we will focus on the numerical approximations of the Volterra integral equation of the second with Carleman kernel of the form:

$$
u(x)=f(x)+\lambda \int_{0}^{x}(|x-t|)^{-v} u(t) \mathrm{d} t
$$




$$
0 \leq v<1,0 \leq t<x,
$$

with weak singularity and $u(t)$ is a continuous function.

The paper is organized as follows: in Section 2, we present various numerical techniques namely: Toeplitz Matrix method, Product Nystrom method, and Sinc-Collocation method for solving Volte-rra integral equation with Carleman kernel, the solutions of two numerical examples are presented in Section 3. Conclusion is drawn in Section 4.

\section{Numerical Schemes}

In this section, we will present the following numerical schemes for solving Volterra integral equation with Carleman kernel:

\subsection{Product Nystrom Method}

To use the product Nystrom method as a numerical technique [3],[9],[14],[24], we consider the integral equation

$$
u(x)=f(x)+\lambda \int_{0}^{x} g(|x-t|) u(t) d t,
$$

so the kernel has the form

$$
g(x, t)=|x-t|^{-v},
$$

we often factor out the singularity in $g$ by writing

$$
g(x, t)=p(x, t) \widetilde{g}(x, t),
$$

where $p(x, t)$ is "badly behaved function and $\widetilde{g}(x, t)$ is "well behaved function. By a suitable Lagrange interpolation polynomial, equation (2.1) can be written as:

$$
u\left(x_{i}\right)=f\left(x_{i}\right)+\lambda \int \sum_{j=1}^{N} w_{i, j} g\left(x_{i}, t_{j}\right) u\left(t_{j}\right),
$$

where, $x=x_{i}=t_{i}=t=i h$ and $i=0,1,2, N$ and $0 \leq x_{0} \leq x_{1} \leq x_{2}, \cdots<$ $x_{n}=X$ with $h=X / N$ and $N$ is even and $w_{i j}$ are the weights which can be determined directly. Moreover, we approximate the integral terms by a product integration using Simpsons rule, where $x=x_{i}$,

$$
\int p\left(x_{i}, t\right) \widetilde{g}\left(x_{i}, t\right) u(t) d t=\sum_{j=0}^{\frac{N-2}{2}} \int_{t_{2 j}}^{t_{2 j+2}} p\left(x_{i}, t\right) \widetilde{g}\left(x_{i}, t_{i}\right) u(t) d t,
$$


Hence, we get

$$
\sum_{j=1}^{N} w_{i j} g\left(x_{i}, t_{j}\right) u\left(t_{j}\right)=\sum_{j=0}^{\frac{N-2}{2}} \int_{t_{2 j}}^{t_{2 j+2}} p\left(x_{i}, t\right) \widetilde{g}\left(x_{i}, t_{i}\right) u(t) d t
$$

Approximating the non-singular part $\widetilde{g}(x, t)) u(t)$ by integration over the interval $\left[t_{2 j}, t_{2 j+2}\right]$ using the second degree of Lagrange interpolation polynomial with the points $t_{2 j}, t_{2 j+1}, t_{2 j+2}$ we get

$$
\int p\left(t_{i}, t\right) \widetilde{g}\left(t_{i}, t\right) u(t) d t=\sum_{j=0}^{N} w_{i, j} \widetilde{g}\left(t_{i}, t_{j}\right) u\left(t_{j}\right)
$$

where

$$
\begin{gathered}
w_{i, 0}=\frac{1}{h^{2}} \int_{t_{0}}^{t_{2}} p\left(t_{i}, t\right)\left(t_{1}-t\right)\left(t_{2}-t\right) d t, \\
w_{i, 2 j+1}=\frac{1}{h^{2}} \int_{t_{2 j}}^{t_{2 j+1}} p\left(t_{i}, t\right)\left(t_{2 j}-t\right)\left(t_{2 j+2}-t\right) d t, \\
w_{i, 2 j}=\frac{1}{2 h^{2}} \int_{t_{2 j-2}}^{t_{2 j}} p\left(t_{i}, t\right)\left(t_{2 j-2}-t\right)\left(t_{2 j-1}-t\right) d t, \\
w_{i, N}=\frac{1}{2 h^{2}} \int_{N-2}^{t_{N}} p\left(t_{i}, t\right)\left(t_{N-2}-t\right)\left(t_{N-1}-t\right) d t,
\end{gathered}
$$

or $w_{i, 0}=\beta_{j}\left(t_{i}\right), w_{i, 2 j+1}=2 \gamma_{j+1}\left(t_{i}\right), w_{i, 2 j}=\alpha_{j}\left(t_{i}+\beta_{j+1}\left(t_{i}\right)\right)$, $w_{i, N}=\alpha_{N / 2}\left(t_{i}\right)$ therefore,

$$
\begin{aligned}
\alpha_{j}\left(t_{i}\right) & =\frac{1}{2 h^{2}} \int_{N-2}^{t_{N}}\left|t_{i}-t\right|^{-v}\left(t-t_{2 j-2}\right)\left(t-t_{2 j-1}\right) d t \\
\beta_{j}\left(t_{i}\right) & =\frac{1}{2 h^{2}} \int_{t_{2 j-2}}^{t_{2 j}}\left|t_{i}-t\right|^{-v}\left(t_{2 j-2}-t\right)\left(t_{2 j}-t\right) d t \\
\gamma_{j}\left(t_{i}\right) & =\frac{1}{2 h^{2}} \int_{t_{2 j-2}}^{t_{2 j}}\left|t_{i}-t\right|^{-v}\left(t-t_{2 j-2}\right)\left(t_{2 j}-t\right) d t
\end{aligned}
$$

Therefore, the integral equation (2.1) reduced to the following system of linear algebraic equations:

$$
u\left(x_{i}\right)=f\left(x_{i}\right)+\lambda \sum_{j=0}^{N} w_{i, j} g\left(x_{i}, t_{j}\right) u\left(t_{j}\right), i=0,1,2, \cdots, N
$$


Hence, equation (2.15) yields a system of linear algebraic equations

$$
(I-\lambda W) U=F .
$$

This system (2.16) can be solved directly as:

$$
U=[I-\lambda W]^{-1} F, \text { and }|I-\lambda W| \neq 0 .
$$

Consider the integral equation (2.1) and introduce the change of variable: $t=$ $t_{2 j-2}+\mu h$ where $0 \leq \mu \leq 2$, and if we define

$$
\phi_{i}(z)=\int_{0}^{2} \mu^{i}|z-\mu|^{-v} d \mu, \quad i=0,1,2,
$$

where $z=i-2 j+2$, then the weight in equation becomes:

$$
\begin{gathered}
w_{i, 0}=\frac{h}{2}\left[2 \phi_{0}(z)-3 \phi_{1}(z)+\phi_{2}(z)\right], \quad z=i, \\
w_{i, 2 j+1}=h\left[2 \phi_{1}(z)-\phi_{2}(z)\right], \quad z=i-2 j, \\
w_{i, 2 j}=\frac{h}{2}\left[\phi_{2}(z)-\phi_{1}(z)+2 \phi_{0}(z-2)-3 \phi_{1}(z-2)+\phi_{2}(z-2)\right], \\
z=i-2 j+2, \\
w_{i, N}=\frac{h}{2}\left[\phi_{2}(z)-\phi_{1}(z)\right], \quad z=i-N+2,
\end{gathered}
$$

The values of $\phi_{i}(z), i=0,1,2$ are given by:

$\phi$ Functions

$$
\phi_{0}=\int_{0}^{2}|x-t|^{-v} d t
$$

case(1):

$$
\phi_{0}=\frac{(x-2)^{1-v}}{v-1}-\frac{x^{1-v}}{v-1}
$$

$\operatorname{case}(2)$

$$
\begin{gathered}
\phi_{0}=\frac{(2-x)^{1-v}}{v-1}+\frac{(-x)^{1-v}}{v-1} . \\
\phi_{1}=\int_{0}^{2} t|x-t|^{-v} d t
\end{gathered}
$$


$\operatorname{case}(1)$

$$
\phi_{1}=\frac{-(x+2(1-v))(x-2)^{1-v}+x^{2-v}}{(v-1)(v-2)},
$$

$\operatorname{case}(2)$

$$
\begin{gathered}
\phi_{1}=\frac{(x+2(1-v))(2-x)^{1-v}+(-x)^{2-v}}{(v-1)(v-2)} . \\
\phi_{2}=\int_{0}^{2} t^{2}|x-t|^{-v} d t
\end{gathered}
$$

case(1)

$$
\phi_{2}=\frac{(x-2)^{1-v}\left(4 v^{2}-4 x v-12 v+4 x+2 x^{2}+8\right)-(2 x)^{3-v}}{(v-1)(v-2)(v-3)},
$$

case(2)

$$
\phi_{2}=\frac{-(2-x)^{1-v}\left(4 v^{2}-4 x v-12 v+4 x+2 x^{2}+8\right)+2(-x)^{3-v}}{(v-1)(v-2)(v-3)} .
$$

WMatrix Entries

$$
\begin{gathered}
w_{i, 0}=\frac{h^{1-v}}{2}\left(2 \phi_{0}(x)-3 \phi_{1}(x)+\phi_{2}(x)\right), x=i, \\
w_{i, 0}=\frac{h^{1-v}}{2}\left[\frac{2(x-2)^{1-v}}{v-1}-\frac{2(x)^{1-v}}{v-1}\right. \\
+\frac{3(x+2(1-v))(x-2)^{1-v}}{(v-1)(v-2)}-\frac{3 x^{2-v}}{(v-1)(v-2)} \\
\left.+\frac{(x-2)^{1-v}\left(4 v^{2}-4 x v-12 v+4 x+2 x^{2}+8\right)-(2 x)^{3-v}}{(v-1)(v-2)(v-3)}\right] . \\
w_{i, 2 j+1}=h^{1-v}\left(2 \phi_{1}(x)-\phi_{2}(x)\right), x=i-2 j,
\end{gathered}
$$

case(1)

$$
\begin{aligned}
w_{i, 2 j+1} & =h^{1-v}\left[\frac{-2(x+2(1-v))(x-2)^{1-v}+2 x^{2-v}}{(v-1)(v-2)}\right. \\
& \left.-\frac{(x-2)^{1-v}\left(4 v^{2}-4 x v-12 v+4 x+2 x^{2}+8\right)-(2 x)^{3-v}}{(v-1)(v-2)(v-3)}\right]
\end{aligned}
$$


$\operatorname{case}(2)$

$$
\begin{gathered}
w_{i, 2 j+1}=h^{1-v}\left[\frac{2(x+2(1-v))(2-x)^{1-v}+2(-x)^{2-v}}{(v-1)(v-2)}\right. \\
\left.+\frac{(2-x)^{1-v}\left(4 v^{2}-4 x v-12 v+4 x+2 x^{2}+8\right)+2(-x)^{3-v}}{(v-1)(v-2)(v-3)}\right] \\
w_{i, 2 j}=\frac{h^{1-v}}{2}\left(\phi_{2}(x)-\phi_{1}(x)+2 \phi_{0}(x-2)-3 \phi_{1}(x-2)\right)+\phi_{2}(x-2), \\
x=i-2 j+2,
\end{gathered}
$$

$\operatorname{case}(1)$

$$
\begin{aligned}
w_{i, 2 j} & =\frac{h^{1-v}}{2}\left[\frac{(x-2)^{1-v}\left(4 v^{2}-4 x v-12 v+4 x+2 x^{2}+8\right)}{(v-1)(v-2)(v-3)}\right. \\
& -\frac{2(x)^{3-v}}{(v-1)(v-2)(v-3)}+\frac{(x+2(1-v))(x-2)^{1-v}}{(v-1)(v-2)} \\
& -\frac{(x)^{2-v}}{(v-1)(v-2)}+\frac{2((x-2)-2)^{1-v}-2(x-2)^{1-v}}{v-1} \\
& +\frac{3((x-2)+2(1-v))((x-2)-2)^{1-v}-3(x-2)^{2-v}}{(v-1)(v-2)} \\
& +\frac{((x-2)-2)^{1-v}\left(4 v^{2}-4(x-2) v-12 v+4(x-2)\right)}{(v-1)(v-2)(v-3)} \\
& \left.+\frac{((x-2)-2)^{1-v}\left(2(x-2)^{2}+8\right)-2(x-2)^{3-v}}{(v-1)(v-2)(v-3)}\right]
\end{aligned}
$$


case $(2)$

$$
\begin{aligned}
w_{i, 2 j}= & \frac{h^{1-v}}{2}\left[-\frac{(2-x)^{1-v}\left(4 v^{2}-4 x v-12 v+4 x+2 x^{2}+8\right)}{(v-1)(v-2)(v-3)}\right. \\
& +\frac{2(-x)^{3-v}}{(v-1)(v-2)(v-3)}-\frac{(x+2(1-v))(2-x)^{1-v}}{(v-1)(v-2)} \\
& -\frac{(-x)^{2-v}}{(v-1)(v-2)}+\frac{-2(2-(x-2))^{1-v}+2(-(x-2))^{1-v}}{v-1} \\
& +\frac{-3((x-2)+2(1-v))(2-(x-2))^{1-v}-3(-(x-2))^{2-v}}{(v-1)(v-2)} \\
& +\frac{-(2-(x-2))^{1-v}\left(4 v^{2}-4(x-2) v-12 v+4(x-2)\right)}{(v-1)(v-2)(v-3)} \\
& \left.+\frac{-(2-(x-2))^{1-v}\left(2(x-2)^{2}+8\right)+2(-(x-2))^{3-v}}{(v-1)(v-2)(v-3)}\right] . \\
& \\
w_{i, N}= & \frac{h^{1-v}}{2}\left[\frac{-(2-x)^{1-v}\left(4 v^{2}-4 x v-12 v+4 x+2 x^{2}+8\right)}{(v-1)(v-2)(v-3)}\right. \\
& \left.\frac{2(-x)^{3-v}}{(v-1)(v-2)(v-3)}-\frac{(x+2(1-v))(2-x)^{1-v}-(-x)^{2-v}}{(v-1)(v-2)}\right] .
\end{aligned}
$$

\subsection{Toeplitz Matrix Method}

In this section, we present the Toeplitz matrix method to obtain numerical solution for Volterra integral equation (1.1). The main approach of this method is to obtain a system $N+1$ linear algebraic equations, where $N+1$ is the number of discrimination points. The coefficient matrix is expressed as sum of two matrices, one of them is the Toeplitz matrix and the other is a matrix with zero elements except the first and last column, see [4], [6], [18]. Consider the following Volterra integral equation with discontinuous kernel

$$
u(x)=f(x)+\lambda \int_{0}^{x_{i}} g(|x-t|) u(t) d t, \text { where } 0 \leq v \leq 1,
$$

The integral term in (2.35) can be written as

$$
\int_{0}^{x_{i}}|x-t|^{-v} u(t) d t=\sum_{n=0}^{N-1} \int_{n h}^{n h+h}\left|x_{i}-t\right|^{-v} u(t) d t h=\frac{X}{N},
$$




$$
x_{n}=X,
$$

$x_{i}=i h, i=1,2,3, N, 0 \leq x_{i} \leq X$, we approximate the integral in the righthand side of (2.36) as

$$
\int_{a}^{a+h}\left|x_{i}-t\right|^{-v} u(t) d t=A_{n}\left(x_{i}\right) \phi(a)+B_{n}\left(x_{i}\right) \phi(a+h)+R_{n},
$$

where $A_{n}$ and $B_{n}$ are two arbitrary functions to be determined and $R_{n}$ is the error. Putting $u(t)=1, t$ in (2.37) yields a set of two equations in terms of the two functions $A_{n}$ and $B_{n}$ respectively. If $R_{n}$ is assumed to be negligible, then we can clearly solve this set of equations for $A_{n}$ and $B_{n}$ and obtain

$$
\begin{gathered}
A_{n}\left(x_{i}\right)=\frac{1}{h}\left((n h+h) I\left(x_{i}\right)-J\left(x_{i}\right)\right), \\
B_{n}\left(x_{i}\right)=\frac{1}{h}\left(J\left(x_{i}\right)-n h I\left(x_{i}\right)\right),
\end{gathered}
$$

$I\left(x_{i}\right)$ and $J\left(x_{i}\right)$ have the following forms:

$$
\begin{array}{r}
I\left(x_{i}\right)=\int_{a}^{a+h}\left|x_{i}-t\right|^{-v} u(t) d t=A_{n}\left(x_{i}\right)+B_{n}\left(x_{i}\right), \\
J\left(x_{i}\right)=\int_{a}^{a+h}\left|x_{i}-t\right|^{-v} u(t) d t=a A_{n}\left(x_{i}\right)+(a+h) B_{n}\left(x_{i}\right),
\end{array}
$$

where $a=n h$, then the system of (2.37) becomes:

$$
\begin{aligned}
& \int_{0}^{x_{i}}\left|x_{i}-t\right|^{-v} u(t) d t=\sum_{n=0}^{N-1}\left[A_{n}\left(x_{i}\right) u(n h)+B_{n}\left(x_{i}\right) u(n h+h)\right], \\
&=\sum_{n=0}^{N-1} D_{n}\left(x_{i}\right) u(n h), \\
& D_{n}\left(x_{i}\right) \begin{cases}A_{0}\left(x_{i}\right) & n=0 \\
A_{n}\left(x_{i}\right)+B_{n-1}\left(x_{i}\right) & 0<n<N, \\
B_{N-1}\left(x_{i}\right) & n=N,\end{cases}
\end{aligned}
$$

thus, the integral equation (2.35) becomes

$$
u\left(x_{i}\right)-\lambda \sum_{n=0}^{N} D_{n}\left(x_{i}\right) u(n h)=f\left(x_{i}\right),
$$


If we put $x_{i}=m h$ we get the following system of algebraic equation:

$$
u(m h)-\lambda \sum_{n=0}^{N} D_{m, n}\left(x_{i}\right) u(n h)=f(m h),
$$

the matrix $\left.D_{(} m, n\right)$ may be written as $D_{m, n}=H_{m, n}-E_{m, n}$, where

$$
H_{m, n}=A_{n}(m h)+B_{n-1}(m h), 0 \leq n \leq N,
$$

is a Toeplitz matrix of order $N+1$ and

$$
E_{m, n} \begin{cases}B_{-1}(m h) & n=0, \\ 0 & 0<n<N, \\ A_{N}(m h) & n=N,\end{cases}
$$

represents a matrix of order $N+1$ whose elements are zero except the first and last columns. However, the solution of the system of equations (2.43) can be obtained in the form:

$$
u(m h)=\left[I-\lambda\left(H_{m n}-E_{m n}\right)\right]^{-1} f(m h) .
$$

where $I$ is identity matrix and $\left|I-\lambda\left(H_{m n}-E_{m n}\right)\right| \neq 0$. In the present work we apply this method to solve integral equation (2.1).

case(1): $x>t$

$$
\begin{aligned}
A_{n}(m h) & =(n+1) \frac{\left(h(m-(n+1))^{1-v}-(h(m-n))^{1-v}\right)}{v-1} \\
& +\left(\frac{(m+(1-v)(n+1))\left(h(m-(n+1))^{1-v}\right.}{(v-1)(v-2)}\right. \\
- & \frac{(m+(1-v) n)(h(m-n))^{1-v}}{(v-1)(v-2)}, \\
B_{n}(m h) & =\frac{-(m+(1-v)(n+1))\left(h(m-(n+1))^{1-v}\right.}{(v-1)(v-2)} \\
& +\frac{(m+(1-v) n)(h(m-n))^{1-v}}{(v-1)(v-2)} \\
& -\frac{\left(n(m-(n+1))^{1-v}+\left(n(h(m-n))^{1-v}\right)\right.}{v-1},
\end{aligned}
$$


Then

$$
\begin{aligned}
H_{m n} & =(n+1) \frac{\left(h(m-(n+1))^{1-v}-(h(m-n))^{1-v}\right)}{v-1} \\
& +\frac{(m+(1-v)(n+1))\left(h(m-(n+1))^{1-v}\right.}{(v-1)(v-2)} \\
& -\frac{(m+(1-v) n)(h(m-n))^{1-v}}{(v-1)(v-2)} \\
& -\frac{(m+(1-v) n)(h(m-n))^{1-v}}{(v-1)(v-2)} \\
& +\frac{\left(m+(1-v)(n-1)(h(m-n+1))^{1-v}\right.}{(v-1)(v-2)} \\
& -\frac{\left.(n-1)(m-n))^{1-v}-(n-1)(h(m-n+1))^{1-v}\right)}{v-1} .
\end{aligned}
$$

case(2): $x<t$

$$
\begin{aligned}
A_{n}(m h) & =(n+1) \frac{\left.-\left(h((n+1)-m)^{1-v}\right)+(h(n-m))^{1-v}\right)}{v-1} \\
- & \frac{(m+(1-v)(n+1))\left(h((n+1)-m)^{1-v}\right.}{(v-1)(v-2)} \\
+ & \frac{(m+(1-v) n)(h(n-m))^{1-v}}{(v-1)(v-2)}, \\
B_{n}(m h) & =-\frac{(m+(1-v)(n+1))(h((n+1)-m))^{1-v}}{(v-1)(v-2)} \\
& -\frac{(m+(1-v) n)(h(n-m))^{1-v}}{(v-1)(v-2)} \\
& +\frac{n(h((n+1)-m))^{1-v}-n(h(n-m))^{1-v}}{v-1}
\end{aligned}
$$


Then

$$
\begin{aligned}
H_{m n} & =(n+1) \frac{\left.-(h((n+1)-m))^{1-v}+(h(n-m))^{1-v}\right)}{v-1} \\
& -\frac{(m+(1-v)(n+1))\left(h((n+1)-m)^{1-v}\right.}{(v-1)(v-2)} \\
& +\frac{(m+(1-v) n)(h(n-m))^{1-v}}{(v-1)(v-2)} \\
& +\frac{(m+(1-v) n)(h(n-m))^{1-v}}{(v-1)(v-2)} \\
& -\frac{\left(m+(1-v)(n-1)(h(n-m-1))^{1-v}\right.}{(v-1)(v-2)} \\
& +\frac{(n-1)(h(n-m))^{1-v}-(n-1)(h(n-m-1))^{1-v}}{v-1} .
\end{aligned}
$$

The elements of the first column of the matrix $E_{m n}$ are given by:

$$
\begin{aligned}
E_{m, 0} & =\frac{-(m)(m h)^{1-v}+(m+(1-v))(h(m+1))^{1-v}}{(v-1)(v-2)} \\
& +\frac{(h m)^{1-v}-(h(m+1))^{1-v}}{v-1},
\end{aligned}
$$

While the element of the last column of the matrix $E_{m, n}$ is given by:

$$
\begin{aligned}
E_{m, N} & =(N+1) \frac{-(h((N+1)-m))^{1-v}+(h(N-m))^{1-v}}{v-1} \\
& -\frac{(m+(1-v)(N+1))\left(h((N+1)-m)^{1-v}\right.}{(v-1)(v-2)} \\
& +\frac{(m+(1-v) N)(h(N-m))^{1-v}}{(v-1)(v-2)} .
\end{aligned}
$$

\subsection{Sinc-Collocation method}

The sinc-approximation with single exponential (SE) and double exponential (DE) are considered as one of the efficient methods to solve this type of integral equations [11], [13], [16]. The sinc-approximation for a function $y$ is expressed as:

$$
y(x)=\sum_{j=-N}^{N} y(j h) S(j h)(x), \quad x \in R,
$$


where the sinc-function is given by:

$$
S(j h)(x)=\frac{\sin \pi\left(\left(\frac{x}{h}-j\right)\right.}{\pi\left(\frac{x}{h}-j\right)},
$$

to construct approximation on the interval $L=(a, b)$ we consider tanh transformation and its inverse

$$
\begin{gathered}
t=\phi^{S E}(x)=\frac{b-a}{2} \tanh \left(\frac{x}{2}\right)+\frac{b+a}{2}, \\
x=\phi^{S E}(t)^{-1}=\log \left(\frac{t-a}{b-t}\right),
\end{gathered}
$$

interpolation formula for $y(t)$ over $(a, b)$ is

$$
y(t)=\sum_{j=-N}^{N} y\left(\phi^{S E}(j h)\right) S(j h)\left(\phi^{S E}\right)^{-1}(t) .
$$

Theorem 1. Let $N$ be a positive integer and $0<d<\pi$, then $h$ is given as

$$
h=\sqrt{\frac{\pi d}{\alpha N}}, \quad \alpha=1-v .
$$

The sinc-function is required to be zero at the end points, $t=a$ and $t=b$, which seems to be an impractical assumption. To handle more general cases, we introduce the translation function [11]:

$$
\Gamma[y](t)=y(t)-\left[\left(\frac{b-t}{t-a}\right) y(a)-\left(\frac{t-a}{b-t}\right) y(b)\right],
$$

\section{Single Exponential-Sinc Schemes}

The function can be accurately approximated as

$$
\Gamma[u](t)=\sum_{j=-N}^{N} \Gamma[u]\left(\phi^{S E}(j h)\right) S(j h)\left(\phi^{S E}(t)\right)^{-1},
$$

so, the approximate solution $u(x)$ is considered as

$$
\begin{aligned}
P_{N}^{S E}[u](t) & =u(a) w_{a}(t) \\
& +\sum_{j=-N}^{N} \Gamma[u]\left(\phi^{S E}(j h)\right) S(j h)\left(\phi^{S E}(t)\right)^{-1}+u(b) w_{b}(t),
\end{aligned}
$$


where

$$
\begin{aligned}
& w_{a}(t)=\frac{b-t}{b-a}, \\
& w_{b}(t)=\frac{t-a}{b-a},
\end{aligned}
$$

and $h$ is given in (2.62).

The unknown coefficient should be determined. For this purpose, let us approximate the solution as

$$
u_{N}^{S E}=c_{-N-1} w_{a}(t)+\sum_{j=-N}^{N} c_{j}\left(\phi^{S E}(j h)\right) S(j h)\left(\phi^{S E}(t)\right)^{-1}+c_{N+1} w_{b}(t) .
$$

There are $2 N+3$ unknown coefficients on the right-hand side of (2.68) that should be determined. To determine the unknowns, we apply the collocation method, by setting $t=t_{i}^{S E}$

$$
u_{N}^{S E}\left(t_{i}^{S E}\right)=f\left(t_{i}^{S E}\right)+\int\left|t_{i}^{S E}-x\right|^{-v} u_{N}^{S E}(x) d x
$$

where $t=t_{i}^{S E}, i=-N-1,-N, \cdots, N, N+1$. Also,

$$
t_{i}^{S E}= \begin{cases}a & i=-N-1 \\ \phi^{S E}(i h) & i=-N, \cdots, N \\ b & i=N+1\end{cases}
$$

since the Sinc-Collocation method does not allow any singularity in the interval, we split the integral in (2.69) into two integrals to remove the singularity using $x=t_{i}^{S E}$

$$
\begin{aligned}
\int\left|t_{i}^{S E}-x\right|^{-v} u_{N}^{S E}(x) d x & =\int_{i}^{t_{i}^{S E}}\left(t_{i}^{S E}-x\right)^{-v} u_{N}^{S E}(x) d x \\
& +\int_{t_{i}^{S E}} t_{i}^{S E}\left(x-t_{i}^{S E}\right)^{-v} u_{N}^{S E}(x) d x
\end{aligned}
$$

The first integral can accurately be approximated by $A_{N}^{S E}\left[u_{N}^{S E}\right]\left(t_{i}^{S E}\right)$, the operator $A_{N}^{S E}$ is

$$
A_{N}^{S E}[y](t)=(t-a)^{1-v} h \sum_{m=M}^{N} \frac{y\left(\phi_{a, t}^{S E}(m h)\right)}{\left(1+e^{-m h}\right)\left(1+e^{-m h}\right)^{1-v}},
$$


where $N$ is a positive integer, $h$ as expressed in (2.62). The second integral is given by:

$$
B_{N}^{S E}[y](t)=(b-t)^{1-v} h \sum_{m=M}^{N} \frac{y\left(\phi_{t, b}^{S E}(m h)\right)}{\left(1+e^{-m h}\right)\left(1+e^{-m h}\right)^{1-v}},
$$

if we introduce $K_{N}^{S E}=A_{N}^{S E}+B_{N}^{S E}$, we obtain the linear system

$$
\begin{aligned}
& \left(E_{N}^{S E}-K_{N}^{S E}\right) c_{n}=f_{N}^{S E}, \\
& c_{n}=\left[c_{-N-1}, c_{-N}, \cdots, c_{N}, c_{N+1}\right]^{T}, \\
& f_{n}^{S E}=\left[f(a), f\left(t_{-N}^{S E}\right), \cdots, f\left(t_{N}^{S E}\right), f(b)\right]^{T}, \\
& E n^{S E}=\left[\begin{array}{ccccc}
1 & 0 & \cdots & 0 & 0 \\
w_{a}\left(t_{-N}^{S E}\right) & 1 & & 0 & w_{b}\left(t_{-N}^{S E}\right) \\
\vdots & & \ddots & & \vdots \\
w_{a}\left(t_{N}^{S E}\right) & 0 & & 1 & w_{b}\left(t_{-N}^{S E}\right) \\
0 & 0 & \cdots & 0 & 1
\end{array}\right] \\
& K_{n}^{S E}=\left[\begin{array}{ccccc}
B_{N}^{S E}\left[w_{a}\right](a) & \cdots & B_{N}^{S E}\left[S(j h)\left(\phi^{S E}(.)\right)^{-1}\right](a) & \cdots & B_{N}^{S E}\left[w_{b}\right](a) \\
K_{N}^{S E}\left[w_{a}\right]\left(t_{-N}^{S E}\right) & \cdots & K_{N}^{S E}\left[S(j h)\left(\phi^{S E}(.)\right)^{-1}\right]\left(t_{-N}^{S E}\right) & \cdots & K_{N}^{S E}\left[w_{b}\right]\left(t_{-N}^{S E}\right) \\
\vdots & & \vdots & & \vdots \\
K_{N}^{S E}\left[w_{a}\right]\left(t_{N}^{S E}\right) & \cdots & K_{N}^{S E}\left[S(j h)\left(\phi^{S E}(.)\right)^{-1}\right]\left(t_{N}^{S E}\right) & \cdots & K_{N}^{S E}\left[w_{b}\right]\left(t_{N}^{S E}\right) \\
A_{N}^{S E}\left[w_{a}\right](b) & \cdots & A_{N}^{S E}\left[S(j h)\left(\phi^{S E}(.)\right)^{-1}\right](b) & \cdots & A_{N}^{S E}\left[w_{b}\right](b)
\end{array}\right] \text {, }
\end{aligned}
$$

where $n$ has a dimension $2 N+3$. By finding $c_{n}$ we can approximate the solution $u_{N}^{S E}$ using (2.68).

\section{Numerical Examples and Results}

To examine the accuracy and the efficiency of the purposed numerical methods, we have carried out some numerical experiments. We compare numerical results with exact solutions using absolute error (see Tables 1 and 2). Further comparison between the approximate solutions and the exact solutions can be seen in Figures 1 and 2 for fixed $X=0.9$.

Example 1. The Volterra integral equation with Carleman kernel

$$
u(x)=x^{2}+\frac{27}{4000} x^{\frac{8}{3}}-0.01 \int_{0}^{x}|x-t|^{-\frac{1}{3}} u(t) d t,
$$

has the exact solution: $u(x)=x^{2}$. 
Now, the results of Example 1 are depicted in Table 1. It includes the exact and numerical solutions of the proposed techniques and the absolute error between the exact and numerical solutions for each one. Moreover, Figure 1 (a, b, c) compares the exact solution and the approximate solution for the Product Nystrom, Toeplitz Matrix and Sinc- Collocation methods respectively for fixed $X=0.9$.

Table 1: The error resulted for example (3.1) where $X=0.9, N=$ 50.

\begin{tabular}{|c|c|c|c|c|c|c|c|}
\hline$x_{i}$ & $\begin{array}{c}\text { Exact. } \\
\text { Sol. }\end{array}$ & $\begin{array}{c}\text { Nyst. } \\
\text { Sol. }\end{array}$ & $\begin{array}{c}\text { Toepl. } \\
\text { Sol. }\end{array}$ & $\begin{array}{c}\text { Collocat. } \\
\text { Sol. }\end{array}$ & $\begin{array}{c}\text { Error } \\
\text { Nyst. }\end{array}$ & $\begin{array}{c}\text { Error } \\
\text { Toepl. }\end{array}$ & $\begin{array}{c}\text { Error } \\
\text { Collocat. }\end{array}$ \\
\hline 0.00 & 0.0000 & 0.002904 & 0.002906 & 0.004593 & 0.002904 & 0.002906 & 0.004593 \\
0.09 & 0.0081 & 0.011205 & 0.011206 & 0.012900 & 0.003105 & 0.003106 & 0.004800 \\
0.18 & 0.0324 & 0.035827 & 0.035822 & 0.037310 & 0.003423 & 0.003422 & 0.004910 \\
0.27 & 0.0729 & 0.076791 & 0.076788 & 0.076460 & 0.003891 & 0.003888 & 0.003560 \\
0.36 & 0.1296 & 0.134126 & 0.134122 & 0.128260 & 0.004526 & 0.004522 & 0.001340 \\
0.45 & 0.2025 & 0.207839 & 0.207830 & 0.198838 & 0.005339 & 0.005330 & 0.003662 \\
0.54 & 0.2916 & 0.297918 & 0.297909 & 0.292072 & 0.006318 & 0.006309 & 0.000472 \\
0.63 & 0.3969 & 0.404354 & 0.404337 & 0.404060 & 0.007454 & 0.007437 & 0.007160 \\
0.72 & 0.5184 & 0.527080 & 0.527063 & 0.528628 & 0.008680 & 0.008663 & 0.010228 \\
0.81 & 0.6561 & 0.665986 & 0.665957 & 0.667756 & 0.009886 & 0.009857 & 0.011656 \\
0.90 & 0.8100 & 0.820289 & 0.822853 & 0.821979 & 0.010289 & 0.012853 & 0.011979 \\
\hline
\end{tabular}

Example 2. The following Volterra integral equation with Carleman kernel

$$
u(x)=x+\frac{4}{300} x^{\frac{3}{2}}-0.01 \int_{0}^{x}|x-t|^{-\frac{1}{2}} u(t) d t,
$$

has the exact solution: $u(x)=x$.

Performing numerical schemes (2.1), (2.2) and (2.3) respectively we obtain the results depicted in Table 2 and shown in Figure 2 (a, b, c).

\section{Conclusion}

In this article three numerical schemes, namely: Product Nystrom, Toeplitz Matrix and Sinc-Collocation methods have been investigated and implemented to obtain an approximate solution of the linear Volterra integral equation of the second kind with Carleman kernel. The numerical results show clearly that all proposed numerical schemes provide good approximate solutions to 


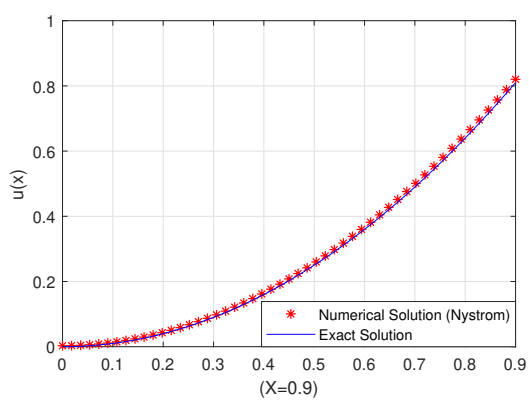

(a)

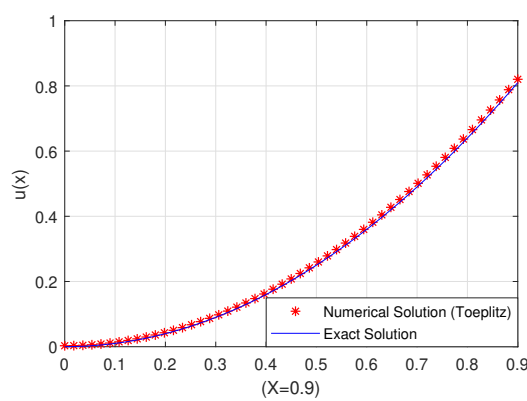

(b)

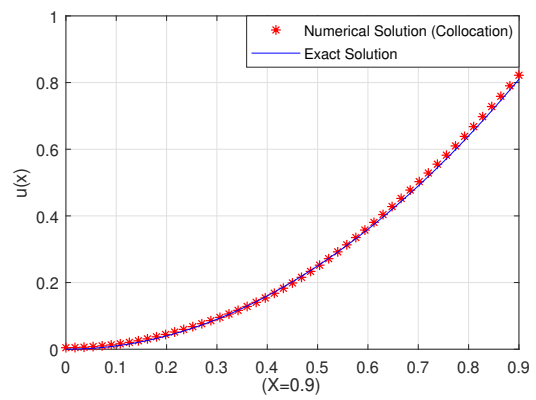

(c)

Figure 1: The exact solution and the approximate solution for Example 1 using (a) the Product Nystrom, (b) Toeplitz Matrix and (c) Sinc-Collocation methods

the Volterra integral equation with Carleman kernel. According to the test numerical examples one can observe from Tables 1 and 2 and Figures 1 and 2 that the product Nystrom method and the Toeplitz matrix method are more effective in comparison with the Sinc-Collocation method.

\section{Acknowledgement}

This manuscript is based on the Master Thesis of W. Draidi entitled "Numerical Techniques for Solving Integral Equations with Carleman Kernel", An-Najah National University, 2017 and supervised by Prof. Naji Qatanani. 
Table 2: The error resulted for example (3.1) where $X=0.9, N=$ 50 .

\begin{tabular}{|c|c|c|c|c|c|c|c|}
\hline$x_{i}$ & $\begin{array}{c}\text { Exact. } \\
\text { Sol. }\end{array}$ & $\begin{array}{c}\text { Nyst. } \\
\text { Sol. }\end{array}$ & $\begin{array}{c}\text { Toepl. } \\
\text { Sol. }\end{array}$ & $\begin{array}{c}\text { Collocat. } \\
\text { Sol. }\end{array}$ & $\begin{array}{c}\text { Error } \\
\text { Nyst. }\end{array}$ & $\begin{array}{c}\text { Error } \\
\text { Toepl. }\end{array}$ & $\begin{array}{c}\text { Error } \\
\text { Collocat. }\end{array}$ \\
\hline 0.00 & 0.00 & 0.005929 & 0.005932 & 0.005914 & 0.005929 & 0.005932 & 0.005914 \\
0.09 & 0.09 & 0.097404 & 0.097387 & 0.097498 & 0.007404 & 0.007387 & 0.007498 \\
0.18 & 0.18 & 0.189322 & 0.189291 & 0.189468 & 0.009322 & 0.009291 & 0.009468 \\
0.27 & 0.27 & 0.281478 & 0.281427 & 0.281802 & 0.01148 & 0.011427 & 0.011802 \\
0.36 & 0.36 & 0.373761 & 0.373701 & 0.374390 & 0.013761 & 0.013701 & 0.014390 \\
0.45 & 0.45 & 0.466123 & 0.466039 & 0.466929 & 0.016123 & 0.016039 & 0.016929 \\
0.54 & 0.54 & 0.558463 & 0.558372 & 0.559241 & 0.018463 & 0.018372 & 0.019241 \\
0.63 & 0.63 & 0.650728 & 0.650611 & 0.651347 & 0.020728 & 0.020611 & 0.021347 \\
0.72 & 0.72 & 0.742737 & 0.742617 & 0.743340 & 0.022737 & 0.022617 & 0.023340 \\
0.81 & 0.81 & 0.834226 & 0.834077 & 0.834929 & 0.024226 & 0.024077 & 0.024929 \\
0.90 & 0.90 & 0.928403 & 0.927944 & 0.923101 & 0.028403 & 0.027944 & 0.023101 \\
\hline
\end{tabular}

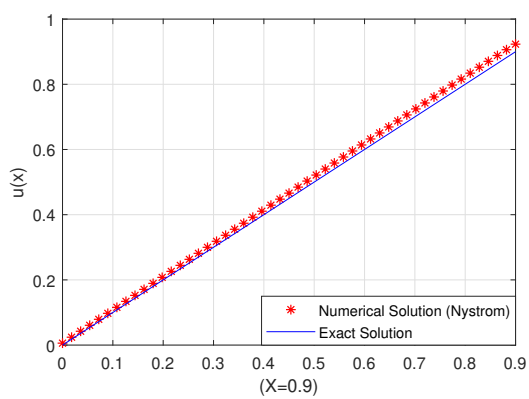

(a)

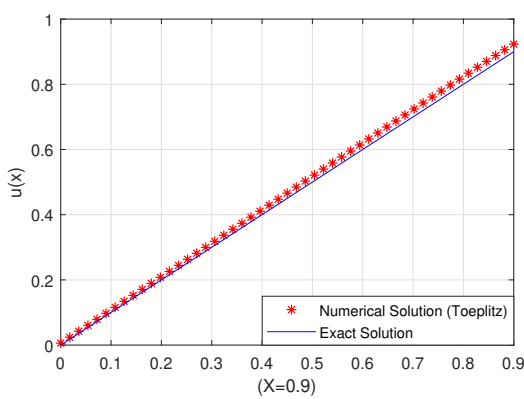

(b)

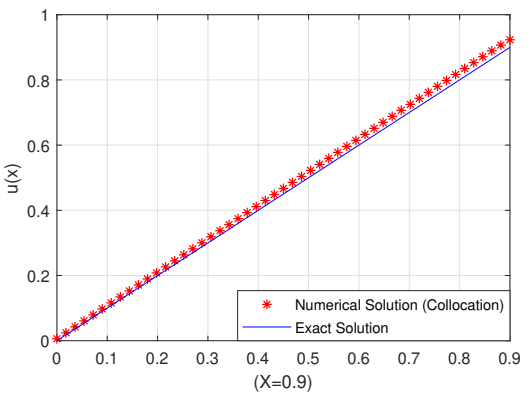

(c)

Figure 2: The exact solution and the approximate solution for Example 2 using (a) the Product Nystrom, (b) Toeplitz Matrix and (c) Sinc-Collocation methods 


\section{References}

[1] J. Abdalkhani, A numerical approach to the solution of Abel's integral equation of the second kind with non-smooth solution, J. Comput. Appl. Math., 29 (1990), 249-355.

[2] M. Abdou, Fredholm-Volterra integral equation and generalized potential kernel, J. Appl. Math. Comput., 131 (2003), 81-94.

[3] M. Abdou, Fredholm-Volterra integral equation with singular kernel, $J$. Appl. Math. Comput., 137 (2003), 231-243.

[4] M. Abdou and G. AbedAlkader, Mixed type of Fredholm Volterra integral equation, Le Matematiche, 60 (2005), 41-58.

[5] M. Abdou and N. Ezzledin, Krein's method with certain singular kernel for solving the integral equation of the first kind, Periodical Math., (2001), 143-149.

[6] M. Abdou, K. Mohamad and A. Ismail, Toeplitz matrix and product nystrom methods for solving the singular integral equation, Le Matematiche, 57 (2002), 21-37.

[7] T. Andrei and V. Alexander, Integral Equations, London (1998).

[8] N. Arytiunian, Plane contact problem of the theory of creep, J. Appl. Math. Mech., 3 (1959), 901-924.

[9] K. Atkinson, The Numerical Solution of Integral Equation of Second Kind, Cambridge University Press, Cambridge (1997).

[10] H. Brunner, The Numerical Solution of Integral Equation with Weakly Singular Kernel, Lecture Notes in Math., Springer, Berlin (1984).

[11] H. Brunner, Collocation Method for Volterra Integral and Related Functional Equations, Cambridge University Press, Cambridge (2004).

[12] W. Draidi, Numerical techniques for solving integral equations with Carleman kernel, M.Sc. Thesis, An-Najah National University (2017).

[13] S. Fayazzadeh and M. Lotfi, Collocation method for Fredholm-Volterra integral equation with weakly kernel, Internat. J. of Mathematical Modeling and Computation, 1 (2001), 59-68. 
[14] F. Garifyanov and S. Modina, The Carleman kernel and its applications, Siberian Math. J., 53 (2012), 1011-1020.

[15] I. Graham, Galerkin method for second kind integral equations with singularities, Mathematics of Computation, 39 (1982), 519-533.

[16] H. Guoqiang and K. Hayami, Extrapolation method of iterated collocation solution of two dimensional nonlinear Volterra integral equation, $J$. Comput. Appl. Math., (1990), 249-355.

[17] F. Hendi, Laplace adomian decomposition method for solving the nonlinear Volterra integral equation with weakly kernels, Studies in Nonl. Sci., 2 (2011), 129-134.

[18] F. Hendi, S. Al-hazmi, The nonlinear Volterra integral equation with weakly kernels and toeplitz matrix method, IJRRAS, 3 (2010), 216-232.

[19] J. Kauthen, A survey of singular perturbed Volterra equations, Appl. Num. Math., 24 (1997), 95-114.

[20] R. Khan and H. Bakodah, Adomian decomposition method and its modification for nonlinear Abel's integral equation, Int. J. of Math. Analysis, 7 (2013), 2349-2358.

[21] A. Kilbas and M. Saigo, On solution of nonlinear Abel-Volterra integral equation, J. Math. Anal. Appl., 229 (1999), 41-60.

[22] P. Linz, Analytic and Numerical Methods for Volterra Equations, SIAM, Philadelphia (1985).

[23] N. Muskhelishvili, Singular Integral Equations, Mir Publisher, Moscow (1953).

[24] T. Okayama, T. Matsu and M. Sugihara, Sinc-collocation methods for weakly singular Fredholm integral equations of the second kind, J. Comput. Appl. Math., 234 (2010), 1211-1227.

[25] A. Orsi, Product integration for Volterra integral equations of the second kind with weakly singular kernel, Math. Comp., 65 (1996), 1201-1212.

[26] M. Tong and W. Chew, A higher-order Nystrm scheme for electromagnetic scattering by arbitrarily shaped surfaces, IEEE Antennas and Wireless Propagation Letters, 4 (2005), 277-280. 
[27] M. Tong and W. Chew, Nyström method with edge condition for electromagnetic scattering by 2D open structures, Progress in Electromagnetics Research (PIER), 62 (2006), 49-68.

[28] M. Tong and W. Chew, E-field, H-field, and combined-field based Nystrm method analysis for electromagnetic scattering by complex material bodies, IEEE Trans. on Electromagnetic Compatibility, 52 (2010), 620-628.

[29] M. Tong, Z. Qian and W. Chew, Nyström method solution of volume integral equations for electromagnetic scattering by $3 \mathrm{D}$ penetrable objects, IEEE Trans. on Antennas and Propagation, 58 (2010), 1645-1652. 
